\title{
Some Generalized Difference Sequence Spaces Defined by Orlicz Functions
}

\author{
B.Sivaraman ${ }^{1}$, K.Chandrasekhara $\mathrm{RaO}^{2}$ and K.Vairamanickam ${ }^{1}$ \\ ${ }^{I}$ Department of Mathematics, Krishnasamy College of Engineering \& Technology, Cuddalore - 607109. \\ ${ }^{2}$ Srinivasa Ramanujan Centre SASTRA University, Kumbakonam, India
}

\begin{abstract}
The idea of difference sequence spaces was introduced by Kizmaz [1] and then this subject has been studied and generalized by various mathematicians. In this paper we define some difference sequence spaces by Orlicz space of bounded sequences and establish some inclusion relations. Some properties of these spaces are studied
\end{abstract}

Keywords: Difference sequence, Bounded sequence, Orlicz function.

Subject Classification: 46A45, 40A05, 40C05, 40D05.

\section{Introduction}

A complex sequence, whose $\mathrm{k}^{\text {th }}$ term is $x_{k}$ is denoted by $\left\{x_{k}\right\}$ or simply $x$. Let $\Phi$ be the set of all finite sequences. A sequence $x=\left\{x_{k}\right\}$ is said to be bounded if $\sup _{k}\left|x_{k}\right|<\infty$. The vector space of all bounded sequences will be denoted by $l_{\infty}$.

Throughout the article $\left(l_{\infty}\right)_{M}$ denote the Orlicz space of bounded sequences respectively.

Throughout $m$ denotes an arbitrary positive integer. Kizmaz [1] introduced the notation of difference sequence spaces as follows: $X(\Delta)=\left\{x=\left(x_{k}\right):\left(\Delta x_{k}\right) \in X\right\}$; for $X=l_{\infty}, c, c_{0}$, where $\Delta x=\left(\Delta x_{k}\right)=\left(x_{k}-x_{k+1}\right)$. Later on the notion was generalized by Et and Colak [2] as follows:

$X\left(\Delta^{m}\right)=\left\{x=\left(x_{k}\right):\left(\Delta^{m} x_{k}\right) \in X\right\}$ for $X=l_{\infty}, c, c_{0}$, where $m \in N, \Delta^{0} x=\left(x_{k}\right)$ and

$\Delta^{m} x=\left(\Delta^{m} x_{k}\right)=\left(\Delta^{m-1} x_{k}-\Delta^{m-1} x_{k+1}\right)$

$=\sum_{v=0}^{m}(-1)^{v}\left(\begin{array}{c}m \\ v\end{array}\right) x_{k+v}$ for all $k \in N$

Later on difference sequence spaces have been studied by Et [3], Et and Nuray [4], Colak Et al [5], Isik [6], Altin and Et [7] and many others.

Orlicz [8] used the idea of Orlicz function to construct the space $\left(L^{M}\right)$. Lindenstrauss and Tzafriri [9] investigated Orlicz sequence spaces in more detail, and they proved that every Orlicz sequence space $l_{M}$ contains a subspace isomorphic to $l_{p}(1 \leq p<\infty)$.

An Orlicz function is a function $\mathrm{M}:[0, \infty) \rightarrow[0, \infty)$ which is continuous, non-decreasing and convex with $\mathrm{M}(0)=0, \mathrm{M}(\mathrm{x})>0$, for $\mathrm{x}>0$ and $M(x) \rightarrow \infty$ as $x \rightarrow \infty$. If convexity of Orlicz function $\mathrm{M}$ is replaced by $M(x+y) \leq M(x)+M(y)$, then this function is called modulus function, defined and discussed by Ruckle [10] and Maddox [11].

Lindenstrauss and Tzafriri [9], S.D.Parashar [12] used the idea of Orlicz function to construct Orlicz sequence space

$l_{M}=\left\{x \in w: \sum_{k=1}^{\infty} M\left(\frac{\left|x_{k}\right|}{\rho}\right)<\infty\right.$, for some $\left.\rho>0\right\}$ where $w=\{$ all complex sequences $\}$.

The space $l_{M}$ with the norm $\|x\|=\inf \left\{\rho>0: \sum_{k=1}^{\infty} M\left(\frac{\left|x_{k}\right|}{\rho}\right) \leq 1\right\}$, becomes a Banach space which is called an Orlicz sequence space. For $M(t)=t^{p}, 1 \leq p<\infty$, the spaces $l_{M}$ coincide with the classical sequence space $l_{p}$. Definition 1.1. The space consisting of all those sequences $x$ in $w$ such that $\left\{\operatorname{Sup}_{k}\left[M\left(\frac{\left|x_{k}\right|}{\rho}\right)\right]\right\}<\infty$ as for some arbitrary fixed $\rho>0$ is denoted by $\left(l_{\infty}\right)_{M}$, M being an Orlicz function. In other words $\left\{M\left(\frac{\left|x_{k}\right|}{\rho}\right)\right\}$ is a bounded sequence. $\left(l_{\infty}\right)_{M}$ is called the Orlicz space of bounded sequences. The space $\left(l_{\infty}\right)_{M}$ is a metric space with the metric $d(x, y)=\operatorname{Sup}_{k} M\left[\frac{\left|x_{k}-y_{k}\right|}{\rho}\right]$ for all $x=\left\{x_{k}\right\}$ and $y=\left\{y_{k}\right\}$ in $\left(l_{\infty}\right)_{M}$.

Definition 1.2. If $\mathrm{M}$ is a convex function and $\mathrm{M}(0)=0$, then $\mathrm{M}(\lambda x) \leq \lambda \mathrm{M}(x)$ for all $\lambda$ with $0<\lambda<1$.

Definition 1.3. A sequence space $E$ is said to be solid or normal if $\left(\alpha_{k} x_{k}\right) \in E$ whenever $\left(x_{k}\right) \in E$ and for all sequences of scalars $\left(\alpha_{k}\right)$ with $\left|\alpha_{k}\right| \leq 1$.

Let $p=\left(p_{k}\right)$ be a sequence of positive real numbers with $0<p_{k}<\sup p_{k}=G$ an let $\mathrm{D}=\operatorname{Max}\left(1,2^{\mathrm{G}-1}\right)$. Then for $a_{k}, b_{k} \in C$, the set of complex numbers for all $k \in N$, we have

$$
\left|a_{k}+b_{k}\right|^{p_{k}} \leq D\left\{\left|a_{k}\right|^{p_{k}}+\left|b_{k}\right|^{p_{k}}\right\}
$$


Let $\mathrm{M}$ be an Orlicz function, $\mathrm{X}$ be locally convex Hausdorff topological linear space whose topology is determined by a set $\mathrm{Q}$ of continuous semi norms q. The symbols $l_{\infty}(X)$ denote the space of all bounded sequences defined over $\mathrm{X}$. We define the following sequence spaces:

$$
\left(l_{\infty}\right)_{M}\left(\Delta^{m}, p, q\right)=\left\{x \in l_{\infty}(X): \operatorname{Sup} \sum_{k=1}^{\infty}\left[M\left(q\left(\frac{\Delta^{m} x_{k}}{\rho}\right)\right)\right]^{p_{k}}<\infty, \text { for some } \rho>0\right\}
$$

\section{Main Results}

Theorem 2.1 If $\mathrm{M}$ is an Orlicz function, then $\left(l_{\infty}\right)_{M}\left(\Delta^{m}, p, q\right)$ is a linear set over the set of complex numbers $\mathrm{C}$.

Proof. Let $x, y \in\left(l_{\infty}\right)_{M}\left(\Delta^{m}, p, q\right)$ and $\alpha, \beta \in C$.

In order to prove the result, we need to find some $\rho_{3}$ such that

$\sum_{k=1}^{\infty}\left[M\left(q\left(\frac{\Delta^{m}\left(\alpha x_{k}+\beta y_{k}\right)}{\rho_{3}}\right)\right)\right]^{p_{k}}<\infty$

Since $x, y \in\left(l_{\infty}\right)_{M}\left(\Delta^{m}, p, q\right)$, there exists some positive $\rho_{1}$ and $\rho_{2}$ such that

$$
\begin{aligned}
& \sum_{k=1}^{\infty}\left[M\left(q\left(\frac{\Delta^{m} x_{k}}{\rho_{1}}\right)\right)\right]^{p_{k}}<\infty \\
& \sum_{k=1}^{\infty}\left[M\left(q\left(\frac{\Delta^{m} y_{k}}{\rho_{2}}\right)\right)\right]^{p_{k}}<\infty
\end{aligned}
$$

Define $\rho_{3}=\max \left(2|\alpha| \rho_{1}, 2|\beta| \rho_{2}\right)$

Since $M$ is a non-decreasing and convex function, q seminorm and $\Delta^{m}$ is linear then

$$
\begin{aligned}
\sum_{k=1}^{\infty}\left[M\left(q\left(\frac{\Delta^{m}\left(\alpha x_{k}+\beta y_{k}\right)}{\rho_{3}}\right)\right)\right]^{p_{k}} & \leq \sum_{k=1}^{\infty}\left[M\left(q\left(\frac{\Delta^{m} \alpha x_{k}}{\rho_{3}}\right)+q\left(\frac{\Delta^{m} \beta y_{k}}{\rho_{3}}\right)\right)\right]^{p_{k}} \\
& \leq \sum_{k=1}^{\infty}\left[M\left(q\left(\frac{\Delta^{m} x_{k}}{\rho_{1}}\right)\right)+M\left(q\left(\frac{\Delta^{m} y_{k}}{\rho_{2}}\right)\right)\right]^{p_{k}} \\
& \leq D \sum_{k=1}^{\infty}\left[M\left(q\left(\frac{\Delta^{m} x_{k}}{\rho_{1}}\right)\right)\right]^{p_{k}}+\mathrm{D} \sum_{k=1}^{\infty}\left[M\left(q\left(\frac{\Delta^{m} y_{k}}{\rho_{2}}\right)\right)\right]^{p_{k}} \\
& \leq \infty
\end{aligned}
$$

By (1.2) and (1.3)

$$
\sum_{k=1}^{\infty}\left[M\left(q\left(\frac{\Delta^{m}\left(\alpha x_{k}+\beta y_{k}\right)}{\rho_{3}}\right)\right)\right]^{p_{k}} \leq \infty
$$

So $(\alpha x+\beta y) \in\left(l_{\infty}\right)_{M}\left(\Delta^{m}, p, q\right)$.

Therefore $\left(l_{\infty}\right)_{M}\left(\Delta^{m}, p, q\right)$ is a linear space.

Theorem 2.2 Let $\mathrm{M}_{1}$ and $\mathrm{M}_{2}$ be two Orlicz functions.

Then $\left(l_{\infty}\right)_{M_{1}}\left(\Delta^{m}, p, q\right) \cap\left(l_{\infty}\right)_{M_{2}}\left(\Delta^{m}, p, q\right) \subseteq\left(l_{\infty}\right)_{M_{1}+M_{2}}\left(\left(\Delta^{m}, p, q\right)\right)$.

Proof.

Let $x \in\left(l_{\infty}\right)_{M_{1}}\left(\Delta^{m}, p, q\right) \cap\left(l_{\infty}\right)_{M_{2}}\left(\Delta^{m}, p, q\right)$.

Then there exists $\rho_{1}$ and $\rho_{2}$ such that

$\sum_{k=1}^{\infty}\left[M\left(q\left(\frac{\Delta^{m} x_{k}}{\rho_{1}}\right)\right)\right]^{p_{k}} \leq \infty \quad$ and $\sum_{k=1}^{\infty}\left[M\left(q\left(\frac{\Delta^{m} x_{k}}{\rho_{2}}\right)\right)\right]^{p_{k}} \leq \infty$

Let $\rho=\min \left(\frac{1}{\rho_{1}}, \frac{1}{\rho_{2}}\right)$. Then we have

$\sum_{n=1}^{\infty}\left[\left(l_{\infty}\right)_{M_{1}+M_{2}}\left(q\left(\frac{\Delta^{m} x_{k}}{\rho}\right)\right)\right]^{p_{k}} \leq$

$$
D\left[\sum_{n=1}^{\infty}\left[\left(l_{\infty}\right)_{M_{1}}\left(q\left(\frac{\Delta^{m} x_{k}}{\rho_{1}}\right)\right)\right]^{p_{k}}\right]+D\left[\sum_{n=1}^{\infty}\left[\left(l_{\infty}\right)_{M_{2}}\left(q\left(\frac{\Delta^{m} x_{k}}{\rho_{1}}\right)\right)\right]^{p_{k}}\right]
$$

$$
\leq \infty
$$

Therefore $\sum_{n=1}^{\infty}\left[\left(l_{\infty}\right)_{M_{1}+M_{2}}\left(q\left(\frac{\Delta^{m} x_{k}}{\rho}\right)\right)\right]^{p_{k}} \leq \infty$.

Hence $x \in\left(l_{\infty}\right)_{M_{1}+M_{2}}\left(\left(\Delta^{m}, p, q\right)\right)$.

Thus $\left(l_{\infty}\right)_{M_{1}}\left(\Delta^{m}, p, q\right) \cap\left(l_{\infty}\right)_{M_{2}}\left(\Delta^{m}, p, q\right) \subseteq\left(l_{\infty}\right)_{M_{1}+M_{2}}\left(\left(\Delta^{m}, p, q\right)\right)$.

Theorem 2.3 Let $m \geq 1$. Then we have the following inclusion $\left(l_{\infty}\right)_{M}\left(\Delta^{m-1}, p, q\right) \subseteq\left(l_{\infty}\right)_{M}\left(\Delta^{m}, p, q\right)$. 
Proof.

$\boldsymbol{x} \in\left(l_{\infty}\right)_{M}\left(\Delta^{m-1}, p, q\right)$. Then we have

$$
\sum_{k=1}^{\infty}\left[M\left(q\left(\frac{\Delta^{m-1} x_{k}}{\rho}\right)\right)\right]^{p_{k}}<\infty, \text { for some } \rho>0
$$

Since $\mathrm{M}$ is non-decreasing convex function and $\mathrm{q}$ is seminorm, we have

$$
\begin{aligned}
\sum_{k=1}^{\infty}\left[M\left(q\left(\frac{\Delta^{m} x_{k}}{\rho}\right)\right)\right]^{p_{k}} & \leq \sum_{k=1}^{\infty}\left[M\left(q\left(\frac{\Delta^{m-1} x_{k}-\Delta^{m-1} x_{k+1}}{\rho}\right)\right)\right]^{p_{k}} \\
& \leq D\left\{\sum_{k=1}^{\infty}\left[M\left(q\left(\frac{\Delta^{m-1} x_{k}}{\rho}\right)\right)\right]^{p_{k}}-\sum_{k=1}^{\infty}\left[M\left(q\left(\frac{\Delta^{m-1} x_{k+1}}{\rho}\right)\right)\right]^{p_{k}}\right\} \\
& \leq \infty
\end{aligned}
$$

Therefore $\sum_{k=1}^{\infty}\left[M\left(q\left(\frac{\Delta^{m} x_{k}}{\rho}\right)\right)\right]^{p_{k}} \leq \infty$.

Hence $x \in\left(l_{\infty}\right)_{M}\left(\Delta^{m}, p, q\right)$.

Thus $\left(l_{\infty}\right)_{M}\left(\Delta^{m-1}, p, q\right) \subseteq\left(l_{\infty}\right)_{M}\left(\Delta^{m}, p, q\right)$.

\section{Theorem 2.4}

a) If $p_{k} \leq 1$ for all $k \in N$ then $\left(l_{\infty}\right)_{M}\left(\Delta^{m}, p, q\right) \subset\left(l_{\infty}\right)_{M}\left(\Delta^{m}, q\right)$.

b) If $p_{k} \geq 1$ for all $k \in N$ then $\left(l_{\infty}\right)_{M}\left(\Delta^{m}, q\right) \subset\left(l_{\infty}\right)_{M}\left(\Delta^{m}, p, q\right)$.

\section{Proof.}

Proof for (a) $x \in\left(l_{\infty}\right)_{M}\left(\Delta^{m}, p, q\right)$. Then

$$
\sum_{k=1}^{\infty}\left[M\left(q\left(\frac{\Delta^{m} x_{k}}{\rho}\right)\right)\right]^{p_{k}}<\infty
$$

since $p_{k} \leq 1$,

$\sum_{k=1}^{\infty}\left[M\left(q\left(\frac{\Delta^{m} x_{k}}{\rho}\right)\right)\right] \leq \sum_{k=1}^{\infty}\left[M\left(q\left(\frac{\Delta^{m} x_{k}}{\rho}\right)\right)\right]^{p_{k}}<\infty$

From (4.1) and (4.2) it follows that

$x \in\left(l_{\infty}\right)_{M}\left(\Delta^{m}, q\right)$. Thus $\left(l_{\infty}\right)_{M}\left(\Delta^{m}, p, q\right) \subset\left(l_{\infty}\right)_{M}\left(\Delta^{m}, q\right)$.

Proof for (b) Let $p_{k} \geq 1$ for all $k$ and

Let $\in\left(l_{\infty}\right)_{M}\left(\Delta^{m}, q\right)$. Then

$\sum_{k=1}^{\infty}\left[M\left(q\left(\frac{\Delta^{m} x_{k}}{\rho}\right)\right)\right]<\infty$

Since $1 \leq p_{k} \leq \sup p_{k}<\infty$, we have

$$
\sum_{k=1}^{\infty}\left[M\left(q\left(\frac{\Delta^{m} x_{k}}{\rho}\right)\right)\right]^{p_{k}} \leq \sum_{\substack{k=1 \\<\infty}}^{\infty}\left[M\left(q\left(\frac{\Delta^{m} x_{k}}{\rho}\right)\right)\right]
$$

Therefore $\in\left(l_{\infty}\right)_{M}\left(\Delta^{m}, p, q\right)$.

Thus $\left(l_{\infty}\right)_{M}\left(\Delta^{m}, q\right) \subset\left(l_{\infty}\right)_{M}\left(\Delta^{m}, p, q\right)$.

Theorem 2.5 $l_{\infty} \subset\left(l_{\infty}\right)_{M}\left(\Delta^{m}, p, q\right)$, with the hypothesis that $\sum_{k=1}^{\infty}\left[M\left(q\left(\frac{\Delta^{m} x_{k}}{\rho}\right)\right)\right]^{p_{k}} \leq\left|x_{k}\right|$.

Proof: Let $x \in l_{\infty}$. Then we have the following implication $\left|x_{k}\right|<\infty$

But $\sum_{k=1}^{\infty}\left[M\left(q\left(\frac{\Delta^{m} x_{k}}{\rho}\right)\right)\right]^{p_{k}} \leq\left|x_{k}\right|$.

By our assumption, implies that

$\sum_{k=1}^{\infty}\left[M\left(q\left(\frac{\Delta^{m_{x_{k}}}}{\rho}\right)\right)\right]^{p_{k}}<\infty$

Then $x \in\left(l_{\infty}\right)_{M}\left(\Delta^{m}, p, q\right)$ and $l_{\infty} \subset\left(l_{\infty}\right)_{M}\left(\Delta^{m}, p, q\right)$.

Theorem 2. $6\left(l_{\infty}\right)_{M}\left(\Delta^{m}, p, q\right)$ is solid.

Proof: Let $\left|x_{k}\right| \leq\left|y_{k}\right|$ and Let $y=\left(y_{k}\right) \in\left(l_{\infty}\right)_{M}\left(\Delta^{m}, p, q\right)$

Because $\mathrm{M}$ is non-decreasing

$\sum_{k=1}^{\infty}\left[M\left(q\left(\frac{\Delta^{m} x_{k}}{\rho}\right)\right)\right]^{p_{k}} \leq \sum_{k=1}^{\infty}\left[M\left(q\left(\frac{\Delta^{m} y_{k}}{\rho}\right)\right)\right]^{p_{k}}$

And because $y \in\left(l_{\infty}\right)_{M}\left(\Delta^{m}, p, q\right)$ 
$\sum_{k=1}^{\infty}\left[M\left(q\left(\frac{\Delta^{m} x_{k}}{\rho}\right)\right)\right]^{p_{k}} \in l_{\infty}$

That is $\sum_{k=1}^{\infty}\left[M\left(q\left(\frac{\Delta^{m} y_{k}}{\rho}\right)\right)\right]^{p_{k}}<\infty$ and

$\sum_{k=1}^{\infty}\left[M\left(q\left(\frac{\Delta^{m} x_{k}}{\rho}\right)\right)\right]^{p_{k}}<\infty$

Therefore $x=\left(x_{k}\right) \in\left(l_{\infty}\right)_{M}\left(\Delta^{m}, p, q\right)$ is solid.

\section{References}

[1] H.Kizmaz, On certain sequence spaces, Canad. Math. Bull., 24 (2) (1981), 169-176.

[2] M.Et and R. Colak, On some generalized difference sequence spaces, Soochow J. Math., 21 (4) (1995), 377 - 386.

[3] M. Et, On some topological properties of generalized difference sequence spaces, Int. J. Math. Math. Sci., 24 (11) (2000), 785 791.

[4] M. Et and F. Nuray, $\Delta^{m}$ - Statistical convergence, Indin J. Pure Appl. Math., 32 (6) (2001), 961 - 969.

[5] R. Colak, M. Et and E. Malkowsky, Some Topics of Sequence Spaces, Lecture Notes in Mathematics. Firat University Press, Elazig, Turkey, 2004.

[6[ M. Isik, On statistical convergence of generalized difference sequences, Soochow J. Math., 30 (2) (2004), 197 - 205.

[7] Y. Altin and M. Et, Generalized difference sequence spaces defined by a modulus function in a locally convex space, Soochow J. Math., 31 (2) (2005), $233-243$.

[8] W. Orlicz, Uber Raume ( $\left.L^{M}\right)$, Bull. Int. Acad. Polon. Sci. A (1936), $93-107$.

[9] J. Lindenstrauss and L. Tzafriri, On Orlicz sequence spaces, Israel J. Math., 10 (1971), $379-390$.

[10] W. H. Ruckle, FK spaces in which the sequence of coordinate vector is bounded, Canad. J. Math, 25 (1973), 973 - 978.

[11] I. J. Maddox, Sequence spaces defined by a modulus, Math. Proc. Combridge Philos. Soc., 100 (1) (1967), 161 - 166.

[12] S.D. Parashar and B. Choudhary, Sequence spaces defined by Orlicz functions, Indian J. Pure. Appl. Math, 25 (4) (1994), 419 - 428. 